\title{
Altered controls transforming normal metabolism into carcinogenic
}

\begin{abstract}
We show how normal metabolism can be transformed into a carcinogenic metabolism in various conditions affecting its controls. A chronic GABA deficiency of the endocrine pancreas that perturbs the regulated secretion of insulin and glucagon will for example, change in a different way the metabolism of stem cells and differentiated cells, leading to a metabolic rewiring process that is advantageous for stem cells; such a rewiring is typical for cancer. We also discuss the role of PKC in normal metabolism and cancer, giving indications for correcting the carcinogenic metabolic rewiring process.
\end{abstract}

Keywords: cancer metabolism, GABA, glucagon, insulin, PKC, stem cells, anti-cancer drugs
Volume 7 Issue 2 - 2017

\author{
Maurice Israël \\ CNRS, France
}

Correspondence: Maurice Israel, CNRS, 2 Av. Aristide Briand 91440, Bures sur Yvette, France, Email mauisrael@wanadoo.fr

Received: November 28, 2016 | Published: January 17, 2017
Abbreviations: PKA, protein kinase a; PKB, protein kinase $\mathrm{b}$; PKC, protein kinase c; IGF, insulin like growth factor; MAPK, mitogen-activated protein kinase pathway; PI3 Kinase, phosphatidylinositide 3-kinase pathway ; PK, pyruvate kinase; PDH, pyruvate dehydrogenase; HSL, hormone sensitive lipase; PEPCK, phosphoenolpyruvate carboxykinase; ACC, acetyl-coa, carboxylase; GSK, glycogen synthase kinase 3; AMP Kin, amp-activated kinase; CPI17, c-kinase-activated protein phosphatase-1 (pp1) inhibitor; I1, pp1 phosphatase inhibitor; GAB, gamma aminobutyric acid; cAMP, cyclic AMP; mTOR, mammalian target of rapamycin; OAA, oxaloacetate; PEP, phosphoenolpyruvate; DAG, diacyl glycerol; IP3, inositol 1,4,5 p; PDK, phosphoinositide-dependent protein kinase; PIP2, phosphatidyl inositol $(4,5)$ Bis P; PIP3, phosphatidylinositol $(3,4,5)$ tris $\mathrm{P} ; 2,3 \mathrm{DPG}, 2,3$-diphosphoglycerate

\section{Phosphorylation of key enzymes controls the direction of metabolic pathways}

\section{Mobilization of tissue stores by catabolic hormones}

When blood glucose is low, the release of glucagon from pancreatic alpha cells, elicits the mobilization of glycogen stores and the synthesis of glucose, correcting hypoglycemia. More generally, in starvation when food gets scarce; the mobilization of glycogen, protein and lipid body stores mediated by catabolic hormones: glucagon, epinephrine and cortisol generate nutrients: glucose and ketone bodies, which maintain essential physiological functions. The glucagon receptor is a Gs protein coupled receptor, which stimulates adenylate cyclase and the formation of cAMP. The latter activates PKA and a set of protein kinases that ultimately control the phosphorylation of a set of enzymes in order to orient metabolic pathways toward the production of glucose by synthesis or glycogenolysis; proteolysis provides amino acids, while lipolysis forms fatty acids producing ketone bodies. In gluconeogenesis, pyruvate kinase $(\mathrm{PK})^{1}$ and pyruvate dehydrogenase (PDH) are phosphorylated by their respective kinases and blocked, closing the entry of the Krebs cycle, while cAMP inhibits the formation of an essential allosteric activator of glycolysis (fructose 2-6 bis phosphate), this will orient the pathway in the reverse neoglucogenic direction. Alanine resulting from proteolysis of muscle proteins is transaminated into pyruvate, which is not consumed by $\mathrm{PK}$ and PDH; and is converted to OAA (pyruvate carboxylase) then to PEP by phosphoenolpyruvate carboxykinase (PEPCK) and follows the neoglucogenic route until glucose. In parallel, the hydrolysis of glycogen stores is elicited by the phosphorylation and activation of phosphorylase a, while the synthesis of glycogen by glycogen synthase is blocked by GSK phosphorylation. As for lipolysis, hormone sensitive lipase (HSL) is activated by phosphorylation, fatty acid are produced, transported by the canityl transporter in liver mitochondria and form ketone bodies by beta-oxidation. Ketone bodies may then provide other tissues with acetyl- CoA. In glycolytic red blood cells devoid of mitochondria, hypoglycemia decreases glycolysis, leading to a decrease of 2-3DPG the allosteric competitor for oxygen binding to hemoglobin, less oxygen is delivered to other tissues, this increase the NADH/NAD + ratio, since there is less oxygen to be reduced by electrons. The increase of NADH, is known to inhibit the citrate condensation reaction; leaving more OAA for supporting neoglucogenesis.

\section{The muscular exception}

There is however an exception to the effects of glucagon, or epineprine in the case of striated muscles; in which there is virtually no glucose synthesis by neoglucogenesis, while glycogenolysis and proteolysis take place. The muscle maintains its glycolytic and oxidative glycolysis, which seems to be linked to their function, they have to be ready for escaping some predator. How then the exception operates? Muscle stimulation is associated to charge movement conducted along $\mathrm{T}$ tubules until ryanodine receptors of endoplasmic reticulum sacs in the depth of the muscle; this opens ryanodine receptors releasing calcium in the cytosol, which initiates the contraction. The release of calcium activates a phosphodiesterase that hydrolyses cAMP into AMP. The decrease of cAMP leads to an increase of fructose 2-6 bis phosphate the allosteric activator of glycolysis. Calcium release in the cytosol stimulates PP2B calcineurin phosphatases, which removes the phosphate from an inhibitor of PP1 (Inhibitor I1) thereby activating PP1. The activation of PP1 might then activate other phosphatases (tyrosine phosphatases). This will dephosphorylate and activate PK and PDH opening the entry of the Krebs cycle. In addition, muscle myoglobin will pull in the necessary oxygen and decrease NADH, which increases the citrate condensation reaction.

\section{Synthetic processes elicited by anabolic hormones}

When blood glucose increases, pancreatic beta cells release insulin. This essential anabolic hormone acts on tyrosine kinase receptors that are activated by phosphorylation. They induce a variety of actions 
triggering the mitotic MAP kinase route, and PI3 Kinase survival pathway, the latter phosphorylates membrane phosphoinositol PIP2 into PIP3. This will elicit the activation of Protein kinase B (PKB) also known as AKT; it is recruited in the membrane where it is activated by PDK (a membrane protein kinase). PKB has effects that are opposite to PKA; it inhibits a set of protein kinases and activates phosphatases, leading in fine to the dephosphorylation of the enzymes that were phosphorylated following catabolic glucagon via PKA. Now dephosphorylated PK and PDH open the entry of the Krebs citric acid cycle, the synthesis of glycogen becomes active since glycogen synthase is no longer phosphorylated by its protein kinase GSK. Inactive (dephosphorylated) phosphorylase b stops hydrolyzing glycogen. As for, the synthesis of proteins, it is stimulated via the activation of mTOR and the eukaryotic initiation factor. The synthesis of fatty acids and lipids is also activated; acetyl- CoA carboxylase (ACC) is "ON"; while HSL and lipolysis are switched off. More generally, anabolic hormones: insulin, growth hormone and insulin like growth factor (IGF) will support the synthesis of new molecular building blocks for dividing cell. The entry of glucose in cells via the glucose transporter and glycolysis are boosted. This again depends of an increase of fructose2-6 bis phosphate the allosteric activator of glycolysis. The mechanism behind is a decrease of cAMP, which cancels the inhibition of fructose 2-6 bis phosphate synthesis. The decrease of cAMP is explained by another downstream effect of the insulin receptor activation: the hydrolysis of membrane phosphoinositide (PIP2) via the activation of a phospholipase $\mathrm{C}$, generating diacylglycerol (DAG) and inositol 1, 4, 5, P (IP3). The latter binds to an IP3 receptor of the endoplasmic reticulum, eliciting the release of calcium. We have seen that this activates a phosphodiesterase that converts cAMP into AMP, and that calcium also activates PP2B then PP1 and other phosphatases that dephosphorylate key enzymes (PK or PDH). In addition the increase of cytosolic calcium triggers the membrane incorporation of the glucose transporter, by exocytosis. Glycolysis and the Krebs cycle - citrate condensation reaction are at work. In parallel, the increased glycolysis in red blood cells forms more 2-3 DPG, which releases more $\mathrm{O} 2$. The reduction of $\mathrm{O} 2$ in tissues receiving more oxygen pulls more electrons, dissociating $\mathrm{NADH}$ into $\mathrm{NAD}+$ and $\mathrm{H}+$, opening the citrate condensation reaction that is no longer inhibited by NADH; adjusting the oxygen supply to the glucose available. We shall see below how these opposite metabolic regulations are perturbed and lead to a hybrid metabolic rewiring ${ }^{2}$ starting a carcinogenic process. ${ }^{2-5}$ But we must first analyze the controls that select the source of acetylCoA.

\section{Selection of the acetyl- CoA source}

The source of acetyl- CoA for citrate condensation is regulated. In a first situation the increase of cytosolic calcium activates, a phosphodiesterase decreasing cAMP and activates phosphatases PP2B then PP1 and others that dephosphorylate and activate PK and PDH; as in the case of the muscle exception analyzed above, stimulated glycolysis produces acetyl- CoA. In a second situation after insulinreceptor actions, the IP3 mediated release of calcium is associated to the formation of diacylglycerol (DAG). This "metabolic increase" of calcium, differs from the calcium increase in muscle, elicited by the electrical activity of working muscles. In the case of insulin, the DAG formed will select the source of acetyl- CoA. When the concentration of DAG increases, it stimulates PKC, which induces the formation of another inhibitor (CPI-17) of the phosphatase PP1. The inhibition of PP1 (a serine phosphatase) suppresses the activation of other tyrosine phosphatases (possibly via an intermediate kinase) keeping in fine PK and PDH phosphorylated on tyrosine's and serine's respectively, closing the glycolytic source of acetyl-CoA. Now fatty acids will have to provide acetyl-CoA by beta-oxidation splitting, after being takenup in mitochondria through the fatty acid carnitine transporter. In this situation, AMP is still elevated and activates AMP kinase, which is known to inhibit ACC, and fatty acid synthesis, the resulting low malonyl- CoA intermediate does not inhibit the mitochondrial carnityl transporter of fatty acids, and the source of acetyl- CoA will come from their beta oxidation cleavage. In a third situation when the anabolic effect of insulin takes over and generate lipids and membranes for mitotic cells, fatty acids have to be synthesized, while their splitting by beta oxidation is stopped. The activation of PKC by DAG stimulates AMP deaminase and 5' nucleotidase (this is documented later) leading to a gradual decrease of AMP; the AMP stimulated kinase being no longer activated, the inhibition of ACC is cancelled, opening the synthesis route for fatty acids. In this case, citrate quits the mitochondria, gives back acetyl- CoA in the cytosol, via ATP citrate lyase, then malonyl- CoA by the action of ACC, following the fatty acid synthesis route via fatty acid synthase (FAS). Recall that the malonyl- CoA intermediate formed by ACC inhibits the mitochondria fatty acid carnityl transporter, closing the beta oxidation process and the fatty acid source of acetyl- CoA. It is thus necessary to open back the glycolytic source of acetyl- CoA and to dephosphorylate PK and PDH. It is presumably a decrease of DAG consumed to form lipids and new membranes that will cancel the stimulation of PKC by DAG, and the formation of CPI-17, the PP1 inhibitor, the dephosphorylation of PDH and PK opens back the glycolytic acetyl- CoA source, In tumor cells, this last step is no longer operational; they consequently have to rewire their metabolic pathways in a very special mode to get their acetyl- CoA via ketone bodies formed by other tissues that produce this nutrient for the tumor cells. This gives them a selective advantage since they plunder other cell reserves. This discussion has much to do with the Randel regulation describing reciprocal interactions between glucose and fatty acid metabolism ${ }^{6}$

\section{The glucose sensor of pancreatic beta cells}

The beta cell membrane contains $\mathrm{K}+$ channels that are inhibited by ATP (K ATP). In hyperglycemia, the cell forms more ATP closing K ATP channels, which depolarizes the cells, and opens calcium channels. The increase of cytosolic calcium elicits the release of insulin, but also the co-release of the inhibitory transmitter GABA. Insulin release will correct hyperglycemia, while GABA controls several processes. It will act on GABA B metabotropic auto-receptors on beta cells to terminate the release of insulin by closing the exocytosis release mechanism. GABA will also act on GABA A receptors of alpha cells eliciting a Cl- influx, which hyperpolarizes the alpha cell and inhibits the release of glucagon from alpha cells. In the same way GABA inhibits the release of somatostatin from pancreatic delta cells. Low somatostatin increases growth hormone $(\mathrm{GH})$ and insulin like growth factor (IGF) release. In other words, when anabolic insulin and IGF operate catabolic glucagon is turned off. It is probable that GABA, synthesized by glutamate decarboxylase (GAD) in the cytosol of beta cell, is constantly released, hyperpolarizing neighboring alpha and delta cells. If blood glucose decreases, a lower ATP content opens KATP channels of beta cells that get hyperpolarized and retain GABA, which elicits a relative depolarization of alpha cell and delta cells that will then release glucagon and somatostatin respectively. In sum, when anabolic beta cells release insulin and GABA in hyperglycemia, they turn off the release of catabolic glucagon. In hypoglycemia when insulin release is blocked, the beta cells retain GABA, which elicits a relative depolarization of alpha cells releasing glucagon. 
This GABA selection switch for hormonal release is crucial, since the phosphorylation status of enzymes controlling the direction of metabolic pathways is opposite for anabolic insulin or catabolic glucagon. Imagine the chaos that would take place if glucagon and insulin did not exclude each other. It is precisely what one may fear if there is a failure of the pancreatic GABA control switch that may have many causes: pesticides, inhibitors of GAD, low GAD cofactor vitamin $\mathrm{B} 6$, or anti GAD auto-antibodies that are known to provoke diabetes. ${ }^{7,8}$ As we shall see, a failure of this switch, explains the observed phosphorylation of enzymes in tumors, the metabolic rewiring and advantage gained by tumor cells over differentiated cells.

\section{Consequences of a pancreatic GABA failure}

First on the beta cell itself, a deficient GABA release will no longer terminate via GABA B metabotropic auto-receptors the release of insulin, a residual but sustained insulin leakage will in the long run, desensitize insulin receptors and presumably affect leptin receptors, establishing a link with diabetes type 2 and obesity.

Second, the deficient GABA release fails to inhibit glucagon release, which then takes place in parallel to insulin. For enzymes such as PDH and PK the glucagon effect dominates, they are phosphorylated causing a bottleneck at the entry of the Krebs cycle. In newly formed cells that are not yet resistant to insulin, the anabolic action of insulin will develop in parallel to the glucagon-dependent bottleneck due to the phosphorylation of PK and PDH; other enzymes such as glycogen synthase or those ensuring the synthesis of proteins and lipids respond to the insulin action and are properly dephosphorylated, synthesizing new molecular building blocks for mitotic cells. In addition we have seen that the activation via PKC of a PP1 phosphatase inhibitor (CPI17), affects PP1 and other phosphatases, maintaining the bottleneck due to PK and PDH phosphorylation induced by glucagon. The cells that display this hybrid anabolic-catabolic response are those that have not been exposed to insulin desensitization, for example, new mitotic stem cells that will then rewire their metabolic pathways. In conclusion, differentiated cells resistant to insulin respond selectively to catabolic hormones mobilizing their stores. Whereas, mitotic stem cells display a dual response, with a blocked PDH associated to an increase of fatty acid and lipid synthesis; the subsequent increase of malonyl- CoA blocks their mitochondrial fatty acid carnityl transporter. Hence, the only way for these cells to get their acetyl$\mathrm{CoA}$ is to convert ketone bodies into acetyl- $\mathrm{CoA}$ and thus to live at the expense of differentiated cells resistant to insulin but responding to glucagon, ${ }^{2,7-9}$ this advantage starts their carcinogenic transformation.

\section{From stem cells to tumor cells}

Each organ contains a population of stem cells entering in mitosis for compensating the death of differentiated cells. Normally the mitosis of a stem cell generates two daughters; one differentiates for replacing a dead cell, while the other gives back a stem cell. We have seen that an alteration of GABA in the pancreas beta cells renders differentiated cells resistant to insulin because they are chronically stimulated by a permanent leakage of insulin; they are on the other hand responding to glucagon. New stem cells that have not been desensitized by insulin respond to both insulin and glucagon, rewiring in a hybrid way their metabolic pathways. This gives them a selective advantage over differentiated cells that are now at work for producing the nutrients needed by metabolically rewired stem cells. In such favorable conditions the stem cells divide and do not differentiate, geometrically increasing the population, they fail to replace the differentiated cells in the organ they should have repaired. So let's examine the main features of the rewiring process that gave them the advantage and find a few ways for reversing the situation., ${ }^{2,9}$

\section{Counteracting the metabolic rewiring of tumor cells}

First, we know that tumor cells display an elevated glycolysis interrupted at the phosphorylated M2 form of PK and phosphorylated PDH bottlenecks. This elevated glycolysis indicates that cAMP did not inhibit the synthesis of fructose 2-6 bis phosphate, which stimulates the observed glycolysis. One may then correct the situation by increasing cAMP using PDE inhibitors (rolipram, curcumin, resveratrol). Second, the serine phosphatase phosphatase PP1 that dephosphorylates directly PDH and indirectly PK via other activated tyrosine phosphatases seems to be inhibited in tumor cells; because PKC activated via insulin, elicits the synthesis of the PP1 inhibitor. Moreover, we know that PKC stimulates AMP deaminase and 5 ' nucleotidase that both decrease AMP, ${ }^{10,11}$ which cancels the stimulation of AMP kinase and its inhibitory action on ACC, leading to an elevated synthesis of fatty acids and lipids in mitotic cells. It would thus be useful to inhibit PKC with non-toxic inhibitors, for example mastica the resin from pistacia lentiscus, ${ }^{12}$ sphingosine ${ }^{13}$ aloe vera anthraquinones such as emodin, ${ }^{14-16}$ there are many other inhibitors of PKC enzastaurin. Inhibiting PKC would also decrease the stimulation by PKC of AMP deaminase and 5'nucleotidase, and increase AMP. One may also inhibit AMP hydrolysis with anthraquinones or sulfonic acid derivatives. ${ }^{16,17}$ The resulting increase of AMP kinase stimulated by AMP would inhibit ACC, and the fatty acid synthesis route, depriving mitotic cells from an essential membrane component. ${ }^{18}$ We have earlier seen that this route is fed by the efflux of citrate from mitochondria via ATP citrate lyase and that it is efficient to block this step with hydroxycitrate. ${ }^{9,} 19$ We have also to consider that the most probable source of acetyl- CoA of tumor cells, are ketone bodies formed in other tissues, since in tumor cells, $\mathrm{PDH}$ is blocked by phosphorylation, while beta oxidation is stopped by a malonyl- $\mathrm{CoA}$ inhibition of the fatty acid canityl transporter. It would thus be useful to reactivate PDH by inhibiting protein kinases (dichloroacetate) and help PDH with (lipoic acid) ${ }^{2,9,19}$ One may try inhibitors for the conversion of ketone bodies into acetyl- CoA using thiolase inhibitors, 4-pentenoic acid. ${ }^{20}$ There are also fatty acid synthase (FAS) inhibitors to be tested. We recall that OAA the other product of ATP citrate lyase drives the transaminases in a direction consuming glutamine and alanine while forming pyruvate and lactate. Transamination inhibition by cycloserine deserves to be studied. In the same line the effects of low arginine diets seem useful ${ }^{21}$ together with actions on the urea cycle (via bicarbonate), or on arginase (norvaline) etc... as discussed elsewhere.

Attention should be given to the effect of citrate (it complexes calcium) and inhibits glycolysis, it has been used with encouraging results.$^{22,23}$ Evidently tumor cells are avid for the citrate quitting the mitochondria, to feed the lipid synthesis route via ATP citrate lyase and ACC, the elevated consummation of citrate decreases its concentration and increases glycolysis in tumor cells. Giving citrate seems to decrease glycolysis but may supplement the lipid synthesis pathway; inhibiting ATP citrate lyase, and ACC was consequently suggested. The metabolic model presented depends much of the properties of protein phosphatases that were remarkably reviewed in another context studying synaptic plasticity. ${ }^{24}$

\section{Conclusion}

The metabolic features associated to cancer seem complex at first sight. However, if one analyses the phosphorylation status enzymes, 
it is possible to deduce that the cells involved have been submitted to a dual action of anabolic insulin and catabolic glucagon, which most probably results from an alteration of GABA controls in the endocrine pancreas. The cells that respond to the dual hormonal actions are new stem cells that are not resistant to insulin as differentiated cells. The latter become the nutritional reservoir of stem cells that efficiently rewire their metabolism in the "cancer mode". The rewiring also depends of an elevated phosphodiesterase activity, associated to a poor PP1 phosphatase activity due to the activation of PKC. Indeed PKC controls PP1 inhibition, and its effects on other phosphatases; it also elicits the decrease of cAMP and AMP, boosting the fatty acid and lipid synthesis of mitotic stem cells that plunder differentiated cells. This metabolic advantage leads to a geometric increase of their number, starting a carcinogenic process that will probably select the most aggressive population.

\section{Acknowledgments}

None.

\section{Conflicts of interest}

Author declares there are no conflicts of interest.

\section{References}

1. Mazurek S, Eigenbrodt E. The tumor metabolome. Anticancer Res. 2003;23(2A):1149-1154.

2. Israël M. Signaling and metabolism in cancer: endocrine pancreas deficiency and hybrid anabolism-catabolism, drugs that undo the process. Cancer Therapy. 2014;10:1-12.

3. Warburg O. On the origin of cancer cells. Science. 1956;123(3191):309-314.

4. Warburg O. On respiratory impairment in cancer cells. Science. 1956;124(3215):269-270

5. Icard $\mathrm{P}$, Lincet $\mathrm{H}$. Global view of the biochemical pathways involved in the regulation of metabolism of cancer cells. Biochimica et Biophysica Acta. 2012;1826(2):423-433.

6. Hue L, Taegtmeyer H. The Randle cycle revisited: a new head for an old hat. Am J Physiol Endocrinol Metab. 2009;297(3):E578- E91.

7. Israël M. A possible primary cause of cancer: deficient cellular interactions in endocrine pancreas. Molecular Cancer. 2012;11:63-68.

8. Israël M. A primary cause of cancer: GABA deficiency in endocrine pancreas. Cancer Therapy. 2012;8:171-183.

9. Israël M, Schwartz L. The metabolic advantage of tumor cells. Molecular cancer. 2011;10(70):1-12.
10. Node K, Kitakaze M, Minamino T, et al. Activation of ecto-5'nucleotidase by protein kinase $\mathrm{C}$ and its role in ischaemic tolerance in canine heart. Br J Pharmacol. 1997;120(2):273-81.

11. HohlCM.AMP deaminase in piglet cardiac myocytes: effect on nucleotide metabolism during ischemia. Am J Physiol. 1999;276(5Pt2):H1502H1510.

12. Triantafyllou A, Bikineyeva A, Dikalova A, et al. Anti-inflammatory activity of Chios mastic gum is associated with inhibition of TNF-alpha induced oxidative stress. Nutr J. 2011;10:64.

13. Hannun YA, Bell RM. Regulation of protein kinase $\mathrm{C}$ by sphingosine and lysosphingolipids. ClinChim Acta. 1989;185(3):333-45.

14. Lim KM, Kwon JH, Kim JY, et al. Emodin inhibits tonic tension through suppressing PKCd-mediated inhibition of myosin phosphatase in rat isolated thoracic aorta. Br J Pharmacol. 2014;171(18):4300-4310.

15. Fredenhagen A, Mett H, Meyer T, et al. Protein tyrosine kinase and protein kinase $\mathrm{C}$ inhibition by fungal anthraquinones related to emodin. The Journal of Antibiotics. 1995;48(11):1355-1358.

16. Baqi Y, Lee S, Iqbal J, et al. Development of potent and selective inhibitors of ecto-5'-nucleotidase based on an anthraquinone scaffold. $J$ Med Chem. 2010;53(5):2076-2086.

17. Raza R, Saeed A, Lecka J, et al. Identification of small sulfonic acids as ecto-5'-nucleotidase inhibitors. Med Chem. 2012;8(6):1133-1139.

18. Svensson RU, Parker SJ, Eichner LJ, et al. Inhibition of acetylCoA carboxylase suppresses fatty acid synthesis and tumor growth in non-small-cell lung cancer in preclinical models. Nat Med. 2016;22(10):1108-1119.

19. Schwartz L, Abolhassani M, Guais A, et al. A combination of alpha lipoic acid and calcium hydroxycitrate is efficient against mouse cancer models: preliminary results. Oncol Rep. 2010;23(5):1407-1416.

20. Schultz H. Metabolism of 4-pentenoic acid and inhibition of thiolase by metabolites of 4-pentenoic acid. Biochemistry. 1983;22(8):1827-1832.

21. Wheatley DN, Campbell E. Arginine deprivation, growth inhibition and tumor cell death: Deficient utilization of citrulline by malignant cell. $\mathrm{Br}$ J Cancer. 2003;89(3):573-576.

22. Bucay AH. The biological significance of cancer; mitochondria as a cause of cancer and the inhibition of glycolysis with citrate as a cancer treatment. Medical Hypotheses. 2007;69(4):826-828.

23. Icard $\mathrm{P}$, Lincet $\mathrm{H}$. The central role of citrate in the metabolism of cancer cells. Biochemical research. 2012;23:SI 31-37.

24. Colbran RJ. Protein phosphatases and Calcium/calmodulin-dependent protein kinase-II-dependent synaptic plasticity. The Journal of Neuroscience. 2004;29:8404-8409. 